09,13

\title{
Проявление сильного квадрупольного взаимодействия и особенности в спектрах гигантского комбинационного рассеяния и усиленного гиперкомбинационного рассеяния молекулы 4, 4'-бипиридина
}

\author{
(C) А.В. Головин ${ }^{1}$, А.М. Полуботко 2, Ф \\ ${ }^{1}$ Санкт-Петербургский государственный университет, \\ Санкт-Петербург, Россия \\ ${ }^{2}$ Физико-технический институт им. А.Ф. Иофрфе РАН, \\ Санкт-Петербург, Россия \\ ฯ E-mail: alex.marina@mail.ioffe.ru \\ (Поступила в Редакцию 11 мая 2016 г. \\ В окончательной редакци 26 декабря 2016 г.)
}

\begin{abstract}
Анализируются спектры гигантского комбинационного рассеяния и усиленного гиперкомбинационного рассеяния 4.4'-бипиридина для двух возможных геометрий, описываемых группами симметрии $D_{2}$ и $D_{2 h}$. Указывается на появление достаточно сильных линий, обусловленных колебаниями с единичным неприводимым представлением для обеих возможных конфигураций. Возникновение этих линий в спектре усиленного гиперкомбинационного рассеяния указывает на существование сильного квадрупольного взаимодействия в системе. Кроме того, в спектрах наблюдаются линии, обусловленные как колебаниями с единичными неприводимыми представлениями $A$ или $A_{g}$, так и неприводимыми представлениями $B_{1}$ или $B_{1 u}$. Последние описывают трансформационные свойства компоненты дипольного момента $d_{z}$, перпендикулярной поверхности. Это свойство спектров обусловлено особенностью геометрии молекулы, состоящей из двух слабо связанных бензольных колец. При этом линейные комбинации колебаний колец образуют два почти вырожденных состояния, симметричное и антисимметричное, которые могут быть неразличимы в экспериментальных спектрах. Результат находится в полном соответствии с дипольно-квадрупольной теорией гигантского комбинационного рассеяния и усиленного гиперкомбинационного рассеяния.
\end{abstract}

DOI: 10.21883/FTT.2017.07.44598.176

\section{1. Введение}

Гигантское комбинационное рассеяние (ГКР) и усиленное гиперкомбинационное рассеяние (усиленное гипер-КР) представляют большой интерес, поскольку могут быть мощным средством научных исследований и применяться в физике, химии и биологии. В большей части литературы эти явления объясняются на основе гипотез о существовании так называемых плазмонов и „химического усиления“. Однако такой подход, учитывающий обычно лишь дипольное взаимодействие света с молекулами, не может объяснить появление запрещенных линий в спектрах молекул с достаточно высокой симметрией. Эта трудность обходится естественным образом при учете квадрупольного взаимодействия света с молекулами, возникающего в сильно неоднородных электромагнитных полях, существующих вблизи шероховатой поверхности металла [1]. В усиленном гипер-КР запрещенные линии, обусловленные полносимметричными колебаниями, преобразующимися по единичному неприводимому представлению, наблюдались в молекулах пиразина и феназина [2-4]. В соответствии с дипольно-квадрупольной теорией запрещенные линии должны появляться в спектрах усиленного гипер-КР и в других молекулах с достаточно высокой симметрией [5]. Однако в литературе данные об их появлении в спектрах усиленного гипер-КР других молекул отсутствуют. Здесь мы проинтерпретировали некоторые особенности спек- тров ГКР и усиленного гипер-КР 4, 4'-бипиридина [6]. В настоящее время в литературе существует два мнения относительно возможной геометрии этой молекулы и ее группы симметрии. В работе [7] на основе компьютерных расчетов и фундаментального принципа минимума потенциальной энергии было определено, что группа симметрии 4, 4'-бипиридина, по всей вероятности, есть $D_{2}$, в то время как в основном в литературе считается, что его группа симметрии $D_{2 h}$. Здесь мы продемонстрируем, что наиболее сильные линии в спектрах ГКР и усиленного гипер-КР обусловлены колебаниями либо с единичными неприводимыми представлениями $A$ и $A_{g}$, либо с неприводимыми представлениями $B_{1}$ и $B_{1 u}$ для групп симметрии $D_{2}$ и $D_{2 h}$ соответственно. Два последних неприводимых представления описывают трансформационные свойства компоненты дипольного момента $d_{z}$, перпендикулярной поверхности. Усиление линий, обусловленных колебаниями с единичным неприводимым представлением, связано с сильным квадрупольным взаимодействием с моментами $Q_{x x}, Q_{y y}, Q_{z z}$ или их линейными комбинациями, имеющими постоянный знак, которые называются в нашей теории основными квадрупольными моментами, в то время как усиление линий с неприводимыми представлениями $B_{1}$ и $B_{1 u}$ связано с рассеянием через два основных квадрупольных момента и рассеянием через момент $d_{z}$ в усиленном гипер-КР. Соответственно в ГКР усиление таких линий обусловлено рассеянием через один основной квадрупольный 
момент и рассеянием через момент $d_{z}$. Усиление, связанное с рассеянием через момент $d_{z}$, обусловлено усилением компоненты электрического поля $E_{z}$, также перпендикулярной поверхности. Однако оказывается, что наиболее сильные линии связаны с механизмами усиления обоих типов, поскольку оба типа колебаний почти вырождены и могут вносить вклад в одну и ту же линию, причем эти почти вырожденные колебания могут быть реально неразрешимы в указанном выше эксперименте [6]. Эта ситуация резко отличается от случая пиразина и феназина и обусловлена специфической геометрией молекулы, состоящей из двух слабовзаимодействующих бензольных колец, что приводит к существованию почти вырожденных симметричного и антисимметричного колебательных состояний с очень близкими частотами.

\section{2. Основные положения дипольно-квадрупольной теории}

Дипольно-квадрупольная теория усиленного гипер-КР в полном объеме была опубликована в $[5,8]$. Кроме того, дипольно-квадрупольные теории ГКР и усиленного инфракрасного поглощения, основанные на этой же концепции сильного квадрупольного взаимодействия света с молекулами, опубликованы в монографии [1] (и например в [9]. Поэтому здесь мы описываем только основные результаты этой теории, отсылая читателя к указанным работам для более полного ознакомления. Ее основным положением является представление о существовании так называемого сильного квадрупольного взаимодействия света с молекулами в поверхностных полях, сильно меняющихся в пространстве, которые всегда возникают вблизи шероховатой поверхности металла. Математически эти поля существуют в связи с необходимостью удовлетворить граничным условиям на нерегулярной шероховатой поверхности. Их появление фактически связано с нарушением симметрии пространства вблизи шероховатой поверхности, поскольку характерный масштаб изменения электромагнитного поля в этой области должен быть равен характерному размеру шероховатости, или характерному размеру изменения свойств среды. Гамильтонианы взаимодействия света с молекулой для падающего поля и поля, рассеянного поверхностью, будут иметь вид

$$
\widehat{\mathbf{H}}_{e-r}^{\text {inc }}=\left|\mathbf{E}_{\text {inc }}\right| \frac{\left(\mathbf{e}^{*} \mathbf{f}^{*}\right)_{\text {inc }} \exp \left(i \omega_{\text {inc }} t\right)+\left(\mathbf{e f}_{e}\right)_{\text {inc }} \exp \left(-i \omega_{\text {inc }} t\right)}{2},
$$

$\widetilde{\mathbf{H}}_{e-r}^{\text {scat }}=\left|\mathbf{E}_{\text {scat }}\right| \frac{\left(\mathbf{e}^{*} \mathbf{f}^{*}\right)_{\text {scat }} \exp \left(i \omega_{\text {scat }} t\right)+\left(\mathbf{e f}_{e}\right)_{\text {scat }} \exp \left(-i \omega_{\text {scat }} t\right)}{2}$.

Здесь $\mathbf{E}_{\text {inc }}$ и $\mathbf{E}_{\text {scat }}$ - падающее и рассеянное поля, $\omega_{\text {inc }}$ и $\omega_{\text {scat }}-$ соответствующие частоты, $\mathbf{e}-$ соответствую-

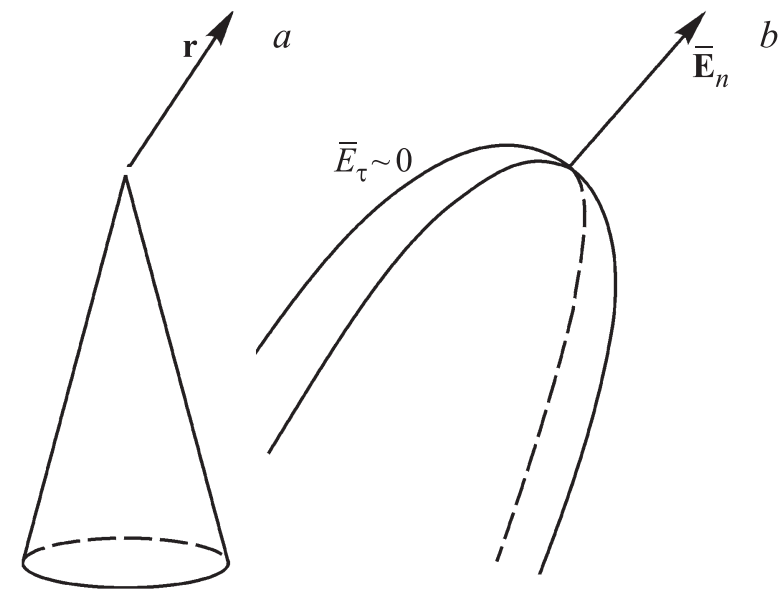

Рис. 1. Модели шероховатостей. $a)-$ конус, $b)-$ более реалистичная модель со скругленной вершиной. В месте максимальной кривизны шероховатости происходит наибольшее усиление нормальной компоненты электрического поля $\overline{\mathbf{E}}_{n}$, в то время как тангенциальная компонента $\overline{\mathbf{E}}_{\tau}$ близка к нулю.

щий вектор поляризации,

$$
f_{e \alpha}=d_{e \alpha}+\frac{1}{2 E_{\alpha}} \sum_{\beta} \frac{\partial E_{\alpha}}{\partial x_{\beta}} Q_{w \alpha \beta}
$$

- $\alpha$-компонента обобщенного вектора взаимодействия света с электронами молекулы,

$$
d_{e \alpha}=\sum_{i} e x_{i \alpha}, \quad Q_{e \alpha \beta}=\sum_{i} e x_{i \alpha} x_{i \beta}
$$

- $\alpha$-компонента вектора дипольного момента и $\alpha \beta$-компонента тензора квадрупольных моментов электронов. Соответственно $x_{i \alpha}$ и $x_{i \beta}-$ декартовы координаты $i$-го электрона. Однако в соответствии с дипольно-квадрупольной теорией только члены гамильтонианов (1), (2), связанные с моментами $Q_{x x}, Q_{y y}, Q_{z z}$ и $d_{z}$, существенны для сильного рассеяния. Эти моменты называются основными моментами $Q_{\text {main }}$ и $d_{\text {main }}[1]$. Указанная значимость этих квадрупольных и дипольного моментов связана с большим усилением компоненты вектора электрического поля $E_{z}$, перпендикулярной поверхности, в областях с большой положительной кривизной, а также с „очень большими“ значениями производных $\frac{\partial E_{\alpha}}{\partial x_{\alpha}}$ по сравнению с их значениями в свободном пространстве. Кроме того, влияние квадрупольного взаимодействия очень сильно из-за некоторых свойств матричных элементов квадрупольных моментов $Q_{x x}, Q_{y y}$ и $Q_{z z}$. Для такой модели шероховатости, как идеально проводящий конус (рис. 1,a), радиальная компонента электрического поля может быть выражена как

$$
E_{r} \sim\left|\mathbf{E}_{0, \text { inc }}\right| C_{0}\left(\frac{l_{1}}{r}\right)^{\beta} .
$$

Здесь $\left|\mathbf{E}_{0 \text {,inc }}\right|-$ амплитуда падающего поля; $C_{0} \sim 1-$ численный коэффициент, $l_{1}-$ характерный размер конуса, например высота; $r$ - радиус-вектор, проведенный из вершины конуса; $0<\beta<1$ и зависит от угла 
при вершине конуса. Коэффициент усиления для чисто дипольного механизма ГКР и усиленного гипер-КР

$$
G_{d} \sim C_{0}^{2 n}\left(\frac{l_{1}}{r}\right)^{2 n \beta},
$$

где $n-$ порядок оптического процесса, $n=2$ для ГКР и $n=3$ для усиленного гипер-КР. Коэффициент усиления для чисто квадрупольного механизма усиления есть

$$
G_{Q} \sim C_{0}^{2 n} \beta^{2 n}\left(\frac{B}{2}\right)^{2 n}\left(\frac{l_{1}}{r}\right)^{2 n \beta}\left(\frac{a}{r}\right)^{2 n} .
$$

Здесь $B \gg 1$ есть некоторый численный коэффициент, $a$ - размер молекулы. Необходимость введения коэффициента $B$ возникает в связи с тем, что матричные элементы квадрупольных моментов с одинаковыми индексами $Q_{x x}, Q_{y y}$ и $Q_{z z}$ значительно больше, нежели считалось до сих пор в квантовой механике. Этот результат связан с тем обстоятельством, что указанные выше квадрупольные моменты являются величинами с постоянным знаком, в то время как дипольные моменты - величины с меняющимся знаком. Поэтому матричные элементы этих квадрупольных моментов значительно больше, чем рассматривалось ранее, и их отношение

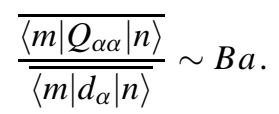

Здесь ранее считалось, что $B \sim 1$. Из (6), (7) можно видеть, что дипольное взаимодействие, связанное с усиленной компонентой электрического поля, перпендикулярной поверхности, и квадрупольное взаимодействие „очень велики“ в малой области в окрестности вершины конуса при $r \rightarrow 0$. Необходимо отметить, что для более реальной модели шероховатости, когда вершина конуса не является идеально острой, а имеет большую, но конечную кривизну (рис. $1, b$ ), электрическое поле на вершине не обращается в бесконечность. Однако электрическое поле и его производные существенно усилены в малой области около вершины, и как дипольное, так и квадрупольное взаимодействие становятся очень большими. В связи с большой ролью квадрупольного взаимодействия в поверхностно-усиленном гипер-КР интенсивность какой-либо линии может определяться несколькими вкладами с различными комбинациями дипольных и квадрупольных моментов (рис. 2). Сечение рассеяния на соответствующих модах колебаний пропорционально сумме этих вкладов [5]

$$
\sigma_{\text {SEHRS }_{S}} \sim \sum_{p}\left|\sum_{f_{1}, f_{2}, f_{3}} T_{(s, p), f_{1}-f_{2}-f_{3}}\right|^{2} d O .
$$

Здесь $f_{1}, f_{2}$ и $f_{3}$ обозначают различные дипольные

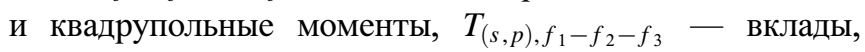
возникающие благодаря различным типам рассеяния, $d O$ - телесный угол. Индексы $(s, p)$ относятся к

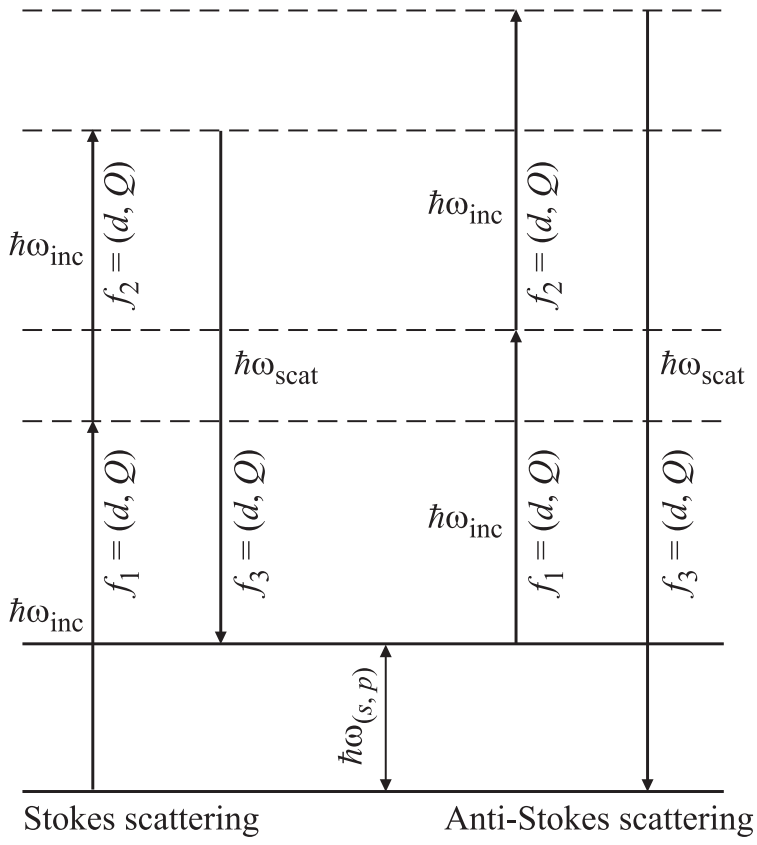

Рис. 2. Диаграмма рассеяния поверхностно-усиленного гипер-КР для стоксова и антистоксова рассеяния. Виртуальное поглощение и испускание могут возникать через различные дипольные и квадрупольные моменты $f_{1}, f_{2}$ и $f_{3}$.

вырожденным колебательным модам. При этом индекс $s$ нумерует группу вырожденных состояний, а индекс $p-$ состояния внутри группы. Вклады $T_{(s, p), f_{1}-f_{2}-f_{3}}$, которые в дальнейшем будем обозначать как $f_{1}-f_{2}-f_{3}$, подчиняются правилам отбора

$$
\Gamma_{(s, p)} \in \Gamma_{f_{1}} \Gamma_{f_{2}} \Gamma_{f_{3}},
$$

где символ Г обозначает неприводимые представления, описывающие трансформационные свойства колебания $(s, p)$ и моментов $f_{1}, f_{2}$ и $f_{3}$. Отметим, однако, что величины вкладов могут значительно отличаться друг от друга. Поэтому для определения интенсивности какой-либо линии часто можно ограничиться только одним, самым сильным вкладом, который и будет определять сечение рассеяния и значение интенсивности. Поскольку квадрупольное взаимодействие с основными квадрупольными моментами может быть наиболее сильным, наиболее усиленными вкладами могут являться вклады типа $Q_{\text {main }}-Q_{\text {main }}-Q_{\text {main }}$, описывающие рассеяние через три основных квадрупольных момента. Так как компонента электрического поля $E_{z}$, перпендикулярная поверхности, тоже усилена, вклады типа $Q_{\text {main }}-Q_{\text {main }}-d_{z}$ будут также усилены, но в меньшей степени, чем предыдущие. Вклады типа $Q_{\text {main }}-d_{z}-d_{z}$ и $d_{z}-d_{z}-d_{z}$ будут тоже усилены, но в меньшей степени, чем предыдущие два типа. Поскольку основные квадрупольные моменты преобразуются по единичному неприводимому представлению, в соответствии с правилами отбора (10) наиболее усиленными будут линии, обусловленные колебаниями, преобразующимися по единичному неприво- 


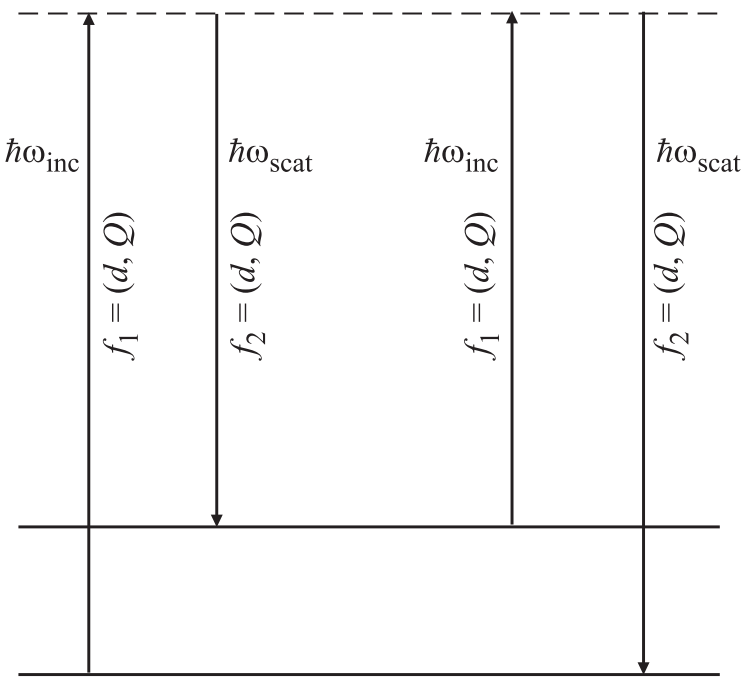

Stokes scattering

Anti-Stokes scattering

Рис. 3. Диаграмма рассеяния ГКР для стоксова и антистоксова рассеяния. Виртуальное поглощение и испускание могут возникать через различные дипольные и квадрупольные моменты $f_{1}$ и $f_{2}$.

димому представлению и по неприводимому представлению, описывающему трансформационные свойства компоненты дипольного момента $d_{z}$. Линии, обусловленные колебаниями, преобразующимися по единичному неприводимому представлению, запрещены в обычном гипер-КР в молекулах, принадлежащих группам с достаточно высокой симметрией. Эта ситуация имеет место, в частности, в молекулах пиразина и феназина, которые принадлежат к группе симметрии $D_{2 h}$ и в которых такие линии наблюдались экспериментально [2-4]. В дальнейшем будем также анализировать спектры инфракрасного поглощения, спектры ГКР и обычного комбинационного рассеяния (КР) 4, 4'-бипиридина. В связи с этим здесь мы также кратко опишем основные результаты теории этих спектров симметричных молекул.

В теории ГКР все особенности, касающиеся влияния сильного квадрупольного взаимодействия света с молекулой, остаются теми же, что и в теории усиленного гипер-КР. Сечение рассеяния ГКР выражается через сумму вкладов

$$
\sigma_{\mathrm{SERS}_{S}} \sim \sum_{p}\left|\sum_{f_{1}, f_{2}} T_{(s, p), f_{1}-f_{2}}\right|^{2} d O .
$$

При этом теперь каждый вклад зависит только от двух дипольных или квадрупольных моментов в соответствии с порядком процесса (рис. 3). Соответствующие вклады удовлетворяют правилам отбора

$$
\Gamma_{(s, p)} \in \Gamma_{f_{1}} \Gamma_{f_{2}} .
$$

Эти вклады будем обозначать как $f_{1}-f_{2}$. Здесь также сечение одной и той же линии может определяться несколькими вкладами. Так как квадрупольное взаимодействие с основными квадрупольными моментами может быть самым сильным, наиболее усиленными вкладами будут те, которые обусловлены рассеянием через два основных квадрупольных момента: $Q_{\text {main }}-Q_{\text {main }}$. Вклады типа $Q_{\text {main }}-d_{z}$ будут также существенно усилены из-за усиления компоненты электрического поля $E_{z}$, перпендикулярной поверхности. Линии, обусловленные преимущественно типом рассеяния $Q_{\text {main }}-Q_{\text {main }}$, определяются полносимметричными колебаниями, преобразующимися по единичному неприводимому представлению. Вследствие того что вклад $d_{z}-d_{z}$ также существенно усилен и обусловлен колебаниями с единичным неприводимым представлением, эти линии не запрещены в ГКР. Однако линии, обусловленные вкладами типа $Q_{\text {main }}-d_{z}$, будут запрещены в ГКР в молекулах с достаточно высокой симметрией, там, где момент $d_{z}$ преобразуется по неприводимому представлению, отличному от единичного. Отметим, что такие линии наблюдаются в очень большом числе симметричных молекул. Как известно, в обычном КР сечение рассеяния и правила отбора формально совпадают с выражениями (11) и (12). Однако $f_{1}$ и $f_{2}$ являются моментами только дипольного типа. Поэтому в обычном КР существуют разрешенные линии, обусловленные колебаниями, преобразующимися по единичному неприводимому представлению, как и в ГКР. В свою очередь в обычном инфракрасном поглощении существуют только линии, обусловленные колебаниями, преобразующимися как компоненты дипольного момента.

\section{3. Особенности спектров ГКР и усиленного гипер-КР 4, 4'-бипиридина с геометрией принадлежащей группе симметрии $\mathrm{D}_{2}$}

Рассмотрим особенности спектров усиленного гипер-КР и ГКР 4, 4'-бипиридина, опубликованных в [6]. В настоящее время существует несколько мнений относительно геометрии и группы симметрии 4, 4'-бипиридина. В работе [7] было определено, что молекула состоит из двух бензольных колец, соединенных между собой через атомы углерода, где противоположные атомы углерода и водорода заменены атомами азота. При этом бензольные кольца повернуты одно относительно другого на угол $38.7^{\circ}$ (рис. 4). Молекула при этом имеет группу симметрии $D_{2}$. Аналогичное мнение было высказано в [10]. Проведя полную оптимизацию геометрии и определив частоты колебаний, используя гибридный функционал B3LYP с базисом 6-31G(d) в программе Gaussian 03 [11], мы пришли к такому же выводу. Кроме того, в литературе распространено мнение, согласно которому, молекула имеет плоскую геометрию и принадлежит к группе симметрии $D_{2 h}$ (рис. 5). Мы рассчитали разницу энергии молекулы с конфигурацией, принадлежащей группе $D_{2 h}$ и группе $D_{2}$. Эта 
Таблица 1. Соотнесение линий в спектрах усиленного гипер-КР, ГКР, КР и инфракрасного поглощения 4, 4'- бипиридина для группы симметрии $D_{2}$ (классификация интенсивностей: оч. сл. - очень слабая, сл. - слабая, ср. - средняя, с. - сильная, оч. с. - очень сильная)

\begin{tabular}{|c|c|c|c|c|c|c|c|}
\hline \multicolumn{4}{|c|}{ Волновые числа, $\mathrm{cm}^{-1}$ (эксперимент) } & \multirow{2}{*}{\multicolumn{2}{|c|}{$\begin{array}{c}\text { Наиболее вероятное } \\
\text { соотнесение с неприводимыми } \\
\text { представлениями (наиболее близкие } \\
\left.\text { значения волновых чисел, } \mathrm{cm}^{-1}\right)\end{array}$}} & \multirow{2}{*}{\multicolumn{2}{|c|}{$\begin{array}{c}\text { Дополнительное возможное } \\
\text { соотнесение с неприводимыми } \\
\text { представлениями вычисленные } \\
\text { в группе симметрии } D_{2}\end{array}$}} \\
\hline \multirow{2}{*}{$\begin{array}{l}\text { Усиленное } \\
\text { гипер-КР }\end{array}$} & \multirow{2}{*}{ ГКР } & \multirow{2}{*}{ КР } & \multirow{2}{*}{$\begin{array}{c}\text { Инфракрасное } \\
\text { поглощение }\end{array}$} & & & & \\
\hline & & & & $A$ & $B_{1}$ & $B_{2}$ & $B_{3}$ \\
\hline 386 сл. & 396 сл. & 388 сл. & $\begin{array}{c}\text { Данные } \\
\text { отсутствуют }\end{array}$ & $A(383)$ & $B_{1}(377)$ & & \\
\hline 477 cp. & & 574 сл. & $\begin{array}{l}499 \text { c. } \\
570 \text { c. }\end{array}$ & & & $B_{2}(570)$ & $\stackrel{?}{B_{3}(500)}$ \\
\hline $\begin{array}{c}628 \text { с. } \\
660 \text { оч. сл. }\end{array}$ & $\begin{array}{l}630 \text { оч. сл. } \\
660 \text { ср. }\end{array}$ & $661 \mathrm{cp}$. & & & & & $\begin{array}{c}? \\
B_{3} \stackrel{(658)}{ }\end{array}$ \\
\hline 736 сл. & 736 оч. сл. & & $\begin{array}{l}674 \text { сл. } \\
742 \text { ср. }\end{array}$ & $A(743)$ & & $\begin{array}{l}B_{2}(670) \\
B_{2}(743)\end{array}$ & \\
\hline 774 сл. & 774 cp. & 767 ср. & 807 оч. с. & & & & $\begin{array}{c}? \\
B_{3}(797)\end{array}$ \\
\hline 864 оч. сл. & $\begin{array}{l}864 \text { оч. сл. } \\
944 \text { ср. }\end{array}$ & 875 сл. & 881 сл. & & $B_{1}(865)$ & $B_{2}(954)$ & $B_{3}(952)$ \\
\hline $\begin{array}{l}1008 \text { ср. } \\
1044 \text { сл. }\end{array}$ & $\begin{array}{l}1018 \mathrm{cp} \\
1038 \mathrm{cp}\end{array}$ & 1011 оч. с. & $\begin{array}{c}990 \mathrm{c} . \\
1040 \mathrm{cp} .\end{array}$ & $A(985)$ & $\begin{array}{c}B_{1}(979) \\
B_{1}(1029)\end{array}$ & & \\
\hline $\begin{array}{l}1064 \text { сл. } \\
1162 \text { сл. }\end{array}$ & 1064 оч. сл. & 1080 сл. & $1076 \mathrm{cp}$ & $A(1073)$ & $B_{1}(1068)$ & & $?$ \\
\hline $1218 \mathrm{cp}$ & $1208 \mathrm{cp}$ & 1224 cp. & 1219 c. & $A(1222)$ & $B_{1}(1218)$ & & \\
\hline $1298 \mathrm{cp}$ & $\begin{array}{l}1300 \text { оч. с. } \\
1438 \text { сл. }\end{array}$ & 1296 оч. с. & & $A(1282)$ & & & $B_{3}(1419)$ \\
\hline $\begin{array}{c}1484 \text { сл. } \\
1516 \text { оч. сл. }\end{array}$ & $\begin{array}{l}1487 \text { оч. сл. } \\
1516 \text { ср. }\end{array}$ & $1514 \mathrm{cp}$ & $\begin{array}{l}1489 \text { c. } \\
1532 \mathrm{cp} .\end{array}$ & $A(1507)$ & $B_{1}(1487)$ & $B_{2}(1543)$ & \\
\hline 1602 оч. с. & $\begin{array}{l}1608 \mathrm{c} . \\
1632 \mathrm{c} .\end{array}$ & $\begin{array}{l}1606 \mathrm{c} . \\
1624 \mathrm{c} .\end{array}$ & 1602 оч. с. & $A(1605)$ & $B_{1}(1600)$ & & $?$ \\
\hline
\end{tabular}

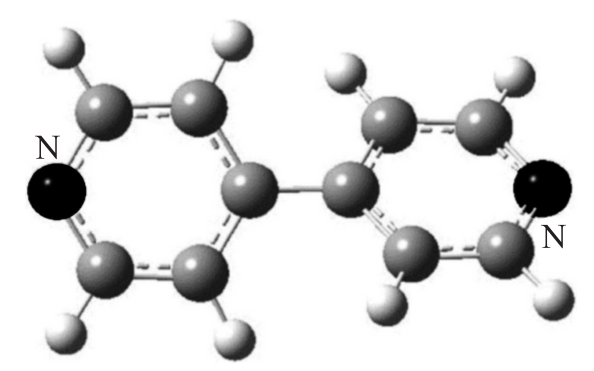

Рис. 4. Структура 4, 4'-бипиридина, имеющего группу симметрии $D_{2}$. Бензольные кольца повернуты относительно друг друга на угол $38.7^{\circ}$.

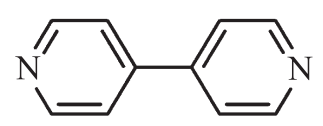

Рис. 5. Структура 4, 4'-бипиридина имеющая группу симметрии $D_{2 h}$. Бензольные кольца лежат в одной плоскости. величина имеет значение $\sim 0.108 \mathrm{eV}$ и по сравнению с $k T \sim 0.026 \mathrm{eV}$ при $T=300 \mathrm{~K}(k-$ постоянная Больцмана) достаточно велика. Это позволяет считать, что молекула принадлежит, скорее всего, к группе $D_{2}$, имеющей четыре неприводимых представления. Выберем ориентацию молекулы по отношению к системе координат таким образом, чтобы ось $z$ проходила через два атома азота. При этом наиболее вероятная ориентация молекулы по отношению к подложке вертикальная, когда она соединена с ней через атом азота, а ось $z$ совпадает по направлению с усиленной компонентой поля $E_{z}$, перпендикулярной поверхности. Мы рассчитали колебательные волновые числа и определили симметрию колебаний молекулы с помощью программы Gaussian 03. Спектры ГКР и усиленного гипер-КР представлены на рис. 6, а соответствующие рассчитанные волновые числа линий и их соотнесение с неприводимыми представлениями собраны в табл. 1. Экспериментальные значения колебательных волновых чисел в спектре усиленного гипер-КР, взятые из [6], а также качественная оценка интенсивности спектральных линий представлены в пер- 


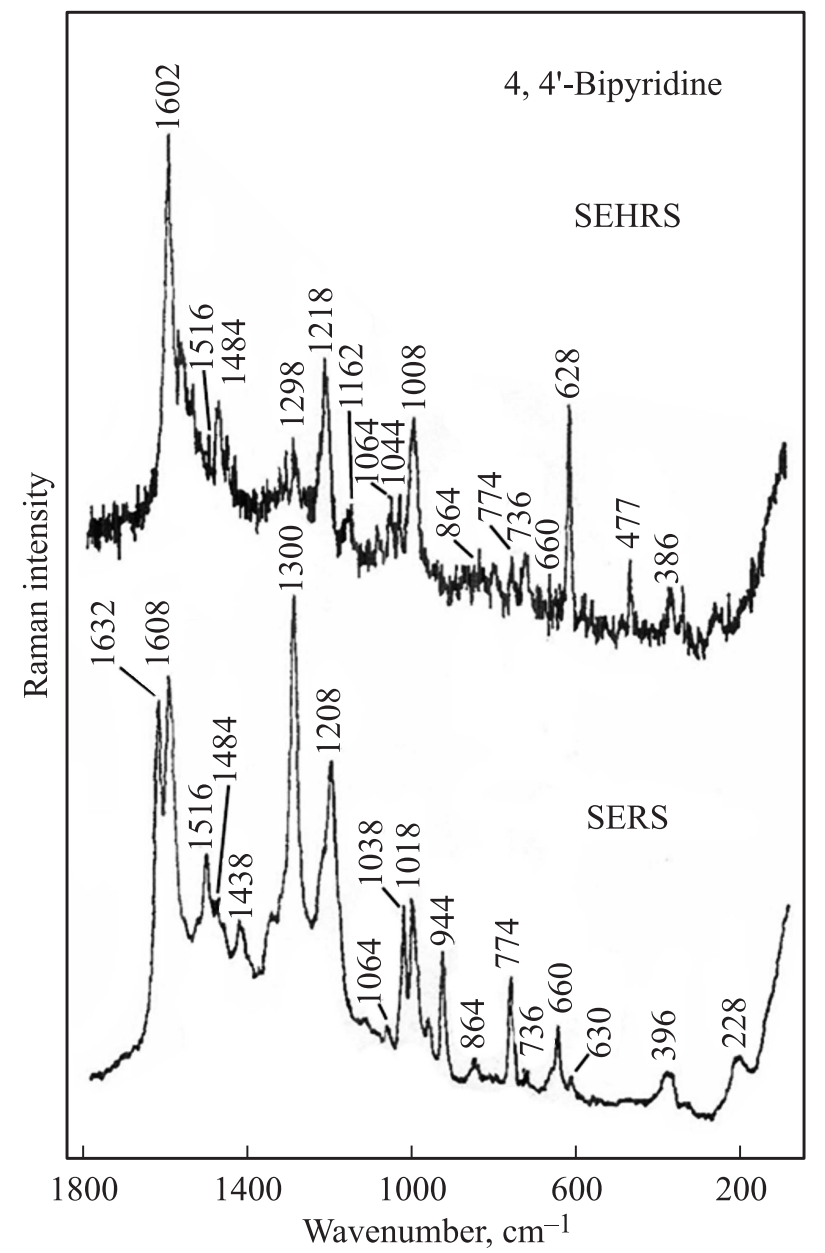

Рис. 6. Спектры усиленного гипер-КР и ГКР 4, 4'-бипиридина. Основа рисунка взята из работы [6].

вом столбце. В трех следующих столбцах представлены экспериментальные значения волновых чисел линий в спектрах ГКР, обычного КР и инфракрасного поглощения $[7,10,12]$. В остальных столбцах, соответствующих возможным неприводимым представлениям, указаны рассчитанные колебательные волновые числа, наиболее близкие к значениям, полученным в эксперименте, и соответствующие наиболее вероятному соотнесению данного колебания. При этом экспериментальные значения, полученные из разных литературных источников, могут различаться не слишком значительно. Однако это несущественно для наших основных выводов.

Основным результатом является то, что в спектре усиленного гипер-КР присутствуют две линии, принадлежащие только единичному неприводимому представлению $A$, со значениями волновых чисел 1298 и $1516 \mathrm{~cm}^{-1}$ и достаточно большой интенсивностью, что указывает на существование сильного квадрупольного взаимодействия в этой системе. Отсутствие этих или очень близких линий в спектре инфракрасного поглощения и их присутствие в спектрах ГКР и обычного КР подтверждают принадлежность этих линий к единичному неприводимому представлению $A$. Указанные линии запрещены в обычном гипер-КР, однако в молекулах с группой симметрии $D_{2}$ они формально разрешены. Это связано с существованием вкладов в рассеяние типа $d_{x}-d_{y}-d_{z}$, обусловленных рассеянием через три дипольных момента $d_{x}, d_{y}$ и $d_{z}$ и колебаниями, преобразующимися по единичному неприводимому представлению $A$. Однако этот вклад должен быть очень мал в случае поверхностно-усиленного гипер-КР, поскольку он определяется тангенциальными компонентами электрического поля $E_{x}$ и $E_{y}$, параллельными поверхности, которые практически равны нулю в связи с ее почти идеальной проводимостью. Как известно, в поверхностной спектроскопии существует так называемый эффект первого слоя (the first layer effect [13]), заключающийся в том, что молекулы, адсорбированные во втором и последующем слоях, усиливают рассеянный свет на порядки слабее, чем молекулы первого слоя. Поэтому вклады типа $d_{x}-d_{y}-d_{z}$ обусловлены лишь молекулами первого слоя и должны быть очень малы. В свою очередь вклады типа $Q_{\text {main }}-Q_{\text {main }}-Q_{\text {main }}$ огромны, и именно они определяют появление сильных линий, обусловленных колебаниями с единичным неприводимым представлением $A$. Эта ситуация аналогична той, которая существует для пиразина и феназина [2-4], и подтверждает теоретические и экспериментальные данные о появлении запрещенных линий в спектрах усиленного гипер-КР [2-5,8].

Другой особенностью спектра усиленного гипер-КР $4,4^{\prime}$-бипиридина является то, что линии с волновыми числами $386,1008,1064,1218$ и $1602 \mathrm{~cm}^{-1}$ могут быть одновременно отнесены к колебаниям как с неприводимым представлением $A$, так и с неприводимым представлением $B_{1}$, описывающим трансформационные свойства компоненты дипольного момента $d_{z}$, перпендикулярной поверхности. Нужно отметить, что в наших расчетах линии с очень малой разницей волновых чисел и симметрией $A$ и $B_{1}$ следуют парами для большого количества колебательных мод. Это обусловлено специфической геометрией молекулы, в которой два бензольных кольца соединены через связь С-C, которая меняет их частоты очень слабо. В результате в молекуле возникают два типа колебательных состояний: симметричные и антисимметричные с неприводимыми представлениями $A$ и $B_{1}$ и очень близкими колебательными частотами. Сходная ситуация описывается в работах $[8,14]$, где подобные пары наблюдались в молекуле транс-1,2-бис(4-пиридил)этилена (trans-1,2-bis(4-peridyle)ethylene). Эта молекула также состоит из двух бензольных колец, слабо связанных через цепочку атомов углерода, и имеет группу симметрии $C_{2 h}$. Аналогично случаю молекулы $4,4^{\prime}$-бипиридина взаимодействие между кольцами может быть слабым, что приводит к появлению двух почти вырожденных симметричного и антисимметричного колебательных состояний, являющихся линейной комбинацией колебаний колец. В случае 4, 4'-бипиридина, так как волновые числа этих состояний очень близки, оба колебания фактически вносят вклад в одну и ту же линию и поэтому могут быть неразличимы в эксперименте. Появление сильных линий с симметрией $B_{1}$ связано с 
Таблица 2. Соотнесение линий в спектрах усиленного гипер-КР, ГКР, КР и инфракрасного поглощения 4, 4'- бипиридина для плоской геометрии и группы симметрии $D_{2 h}$ (классификация интенсивностей: оч. сл. - очень слабая, сл. - слабая, ср. средняя, с. - сильная, оч. с. - очень сильная)

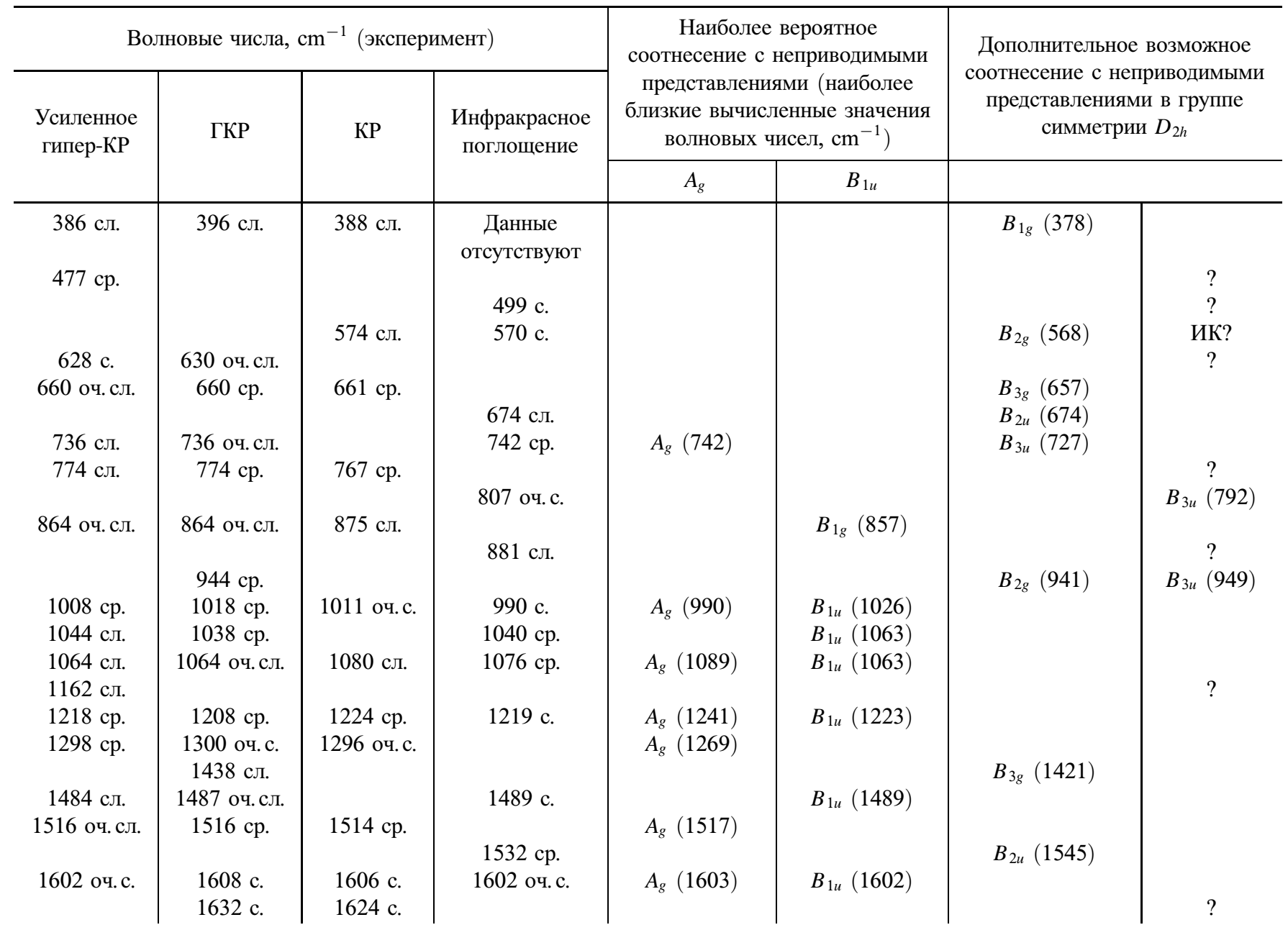

рассеянием типа $Q_{\text {main }}-Q_{\operatorname{main}}-d_{z}$. Тот факт, что линии с волновыми числами $386,1008,1064,1218$ и $1602 \mathrm{~cm}^{-1}$ могут иметь вклады типа $Q_{\text {main }}-Q_{\text {main }}-d_{z}$, подтверждается существованием соответствующих очень близких линий инфракрасного поглощения. Отметим, что в указанных спектрах наблюдается несколько линий с волновыми числами 864, 1044 и $1487 \mathrm{~cm}^{-1}$, обусловленными колебаниями только с неприводимым представлением $B_{1}$. Эти линии проявляются одновременно в спектрах усиленного гипер-КР, ГКР и инфракрасного поглощения и вместе с тем полностью отсутствуют в спектре обычного КР, за исключением, может быть, очень слабой линии $864 \mathrm{~cm}^{-1}$. Этот результат находится в строгом соответствии с нашей теорией. Как известно, детали исследуемых спектров усиленного гипер-КР и ГКР должны сильно зависеть от условий их получения. Однако, к сожалению, в [6] такая информация полностью отсутствует. Кроме того, в силу приближенного характера вычислений установление симметрии колебаний, обусловливающих соответствующие линии, имеет доста- точно большой интервал неопределенности. Поэтому мы не можем определить, к каким колебаниям и с какой симметрией относятся некоторые линии. Это относится к линиям усиленного гипер-КР 477, 628, 774, $1162 \mathrm{~cm}^{-1}$ и линиям ГКР с очень близкими волновыми числами, а также к линии в спектре ГКР $1632 \mathrm{~cm}^{-1}$ и линии при $881 \mathrm{~cm}^{-1}$ в спектре инфракрасного поглощения. Bсе эти случаи обозначены знаком вопроса в последнем столбце табл. 1 Отметим, что линии, относящиеся к неприводимым представлениям $B_{2}$ и $B_{3}$, обусловлены вкладами, содержащими тангенциальные компоненты электрического поля $E_{x}$ и $E_{y}$, которые близки к нулю на поверхности из-за ее высокой проводимости. Поэтому их интенсивность либо очень мала, либо равна нулю. То же самое можно сказать о линиях ГКР с этой же симметрией. Довольно большая интенсивность линий ГКР 660 и $944 \mathrm{~cm}^{-1}$ может быть связана с адсорбцией молекул на шероховатостях атомного масштаба. В этом случае ориентация молекулы по отношению к поверхности может быть произвольной и компонента поля $E_{z}$, 
перпендикулярная поверхности, может иметь все усиленные компоненты поля в системе координат, связанной с молекулой. Такая ситуация может приводить к достаточно высокой интенсивности этих линий. Однако в любом случае можно констатировать, что для рассматриваемой геометрии в спектрах усиленного гипер-КР и ГКР имеются линии, которые могут быть обусловлены колебаниями как с неприводимым представлением $A$, так и с $B_{1}$. Их существование находится в полном соответствии с дипольно-квадрупольной теорией.

\section{4. Анализ спектров усиленного гипер-КР и ГКР для геометрии молекулы с группой симметрии $D_{2 h}$}

Поскольку молекуле 4, 4'-бипиридина часто приписывают группу симметрии $D_{2 h}$, мы провели анализ спектров и для этого случая (табл. 2). При этом линии при 1298 и $1516 \mathrm{~cm}^{-1}$ относятся к колебаниям, преобразующимся по единичному неприводимому представлению $A_{g}$. Они запрещены в обычном гипер-КР. Это указывает на то, что независимо от предполагаемой группы симметрии сильное квадрупольное взаимодействие проявляется в спектре усиленного гипер-КР. Кроме того, в спектрах усиленного гипер-КР и ГКР присутствуют сильные линии, которые могут быть отнесены к колебаниям как с неприводимым представлением $A_{g}$, так и с $B_{1 u}$. Последнее описывает трансформационные свойства дипольного момента $d_{z}$. Это линии с волновыми числами 1008, 1064, 1218 и $1602 \mathrm{~cm}^{-1}$, имеющие фактически те же волновые числа, что и линии с симметрией $A$ и $B_{1}$ в случае принадлежности молекулы к группе симметрии $D_{2}$. Исключение составляет линия $386 \mathrm{~cm}^{-1}$, не имеющая такого соотнесения. В спектрах присутствуют также линии, относящиеся только к неприводимому представлению $B_{1 u}$, описывающему трансформационные свойства момента $d_{z}$. Это линии 1484 и $1044 \mathrm{~cm}^{-1}$. Необходимо отметить, что наше отнесение к неприводимым представлениям $A_{g}$ и $B_{1 u}$ хорошо согласуется с соотнесением, использованным для интерпретации спектров инфракрасного поглощения и обычного КР 4, 4'-бипиридина, опубликованным в [12], что полностью подтверждает наши представления. В силу приближенного характера вычислений, как и при рассмотрении случая группы симметрии $D_{2}$, мы не смогли провести соотнесение некоторых линий. Такие линии обозначены знаком вопроса в последнем столбце табл. 2. Всего у нас возникло восемь таких случаев. Это больше, чем число случаев, возникших при анализе с группой симметрии $D_{2}$, равное шести. Линии, обусловленные колебаниями другой симметрии, отличной от $A_{g}$ и $B_{1 u}$, соответствуют линиям для случая группы $D_{2}$ со следующими правилами соответствия: $B_{1 u}, B_{1 g} \rightarrow B_{1}$, $B_{2 u}, B_{2 g} \rightarrow B_{2}, B_{3 u}, B_{3 g} \rightarrow B_{3}$. Однако здесь существует некоторое исключение для линии $736 \mathrm{~cm}^{-1}$. В целом соотнесение для случая группы $D_{2 h}$, с нашей точки зрения, хуже, чем для случая группы $D_{2}$. Однако основной результат - то, что линии усиленного гипер-КР и ГКР $\left(1008,1064,1218\right.$ и $1602 \mathrm{~cm}^{-1}$ могут быть отнесены как к единичному неприводимому представлению $A_{g}$, так и к неприводимому представлению $B_{1 u}$, - остается тем же. Таким образом, мы иногда не можем выполнить однозначное соотнесение некоторых линий из-за очень близких значений вычисленных волновых чисел колебаний с симметрией $A_{g}$ и $B_{1 u}$, которые следуют парами.

\section{5. Заключение}

Таким образом, анализ спектров усиленного гипер-КР, ГКР, обычного КР и инфракрасного поглощения указывает на существование довольно сильных линий, обусловленных полносимметричными колебаниями, преобразующимися по единичному неприводимому представлению для геометрий молекулы, принадлежащих как к группе симметрии $D_{2}$, так и к группе симметрии $D_{2 h}$. Поскольку вклад типа $d_{x}-d_{y}-d_{z}$ очень мал, можно считать, что их появление есть следствие сильного квадрупольного взаимодействия в спектрах усиленного гипер-КР молекулы 4, 4'-бипиридина. Существование линий, обусловленных колебаниями с неприводимыми представлениями $B_{1}$ или $B_{1 u}$, указывает на присутствие сильного дипольного взаимодействия, связанного с усилением компоненты электрического поля $E_{z}$, перпендикулярной поверхности. Кроме того, несколько линий может быть отнесено к почти вырожденным колебаниям с неприводимыми представлениями $A$ или $B_{1}$ в группе симметрии $D_{2}$ либо к неприводимым представлениям $A_{g}$ или $B_{1 u}$ в группе симметрии $D_{2 h}$. По-видимому, они не могут быть разрешены в эксперименте. Их интенсивность может определяться как типом рассеяния $Q_{\text {main }}-Q_{\text {main }}-Q_{\text {main }}$, так и типом рассеяния $Q_{\text {main }}-Q_{\text {main }}-d_{z}$ в усиленном гипер-КР, а также типом рассеяния $Q_{\text {main }}-Q_{\text {main }}$ или $Q_{\text {main }}-d_{z}$ в ГКР. Это является особенностью спектра усиленного гипер-КР 4, 4'-бипиридина в сравнении с таким же спектром молекулы пиразина [3,4]. Такая ситуация возникает в связи с особенностью геометрии молекулы, которая состоит из двух бензольных колец, колебания которых слабо воздействуют друг на друга и которые формируют симметричное и антисимметричное колебательные состояния с очень близкими частотами. В заключение необходимо отметить, что геометрия 4, 4'-бипиридина с группой симметрии $D_{2}$ более вероятна, поскольку, согласно расчетам, она соответствует минимуму полной энергии молекулы для этой конфигурации и соотнесение линий в этом случае оказывается более точным.

\section{Список литературы}

[1] A.M. Polubotko. The dipole quadrupole theory of surface enhanced Raman scattering. Nova Sci. Publ., Inc., N.Y. (2009). 136 p.

[2] W.H. Li, X.Y. Li, N.T. Yu. Chem. Phys. Lett. 327, 153 (2000).

[3] W.H. Li, X.Y. Li, N.T. Yu. Chem. Phys. Lett. 305, 303 (1999). 
[4] X.Y. Li, Q.J. Huang, V.I. Petrov, Y.T. Xie, Q. Luo, X. Yu, Y.J. Yan. J. Raman Spectrosc. 36, 555 (2005).

[5] A.M. Polubotko, V.P. Smirnov. J. Raman Spectrosc. 43380 (2012).

[6] S. Nie, L.A. Lipscomb, N.T. Yu. Appl. Spectrosc. Rev. 26, 203 (1991).

[7] Z. Zhuang, J. Cheng, X. Wang, B. Zhao, X. Han, Y. Luo. Spectrochim. Acta A 67, 509 (2007).

[8] А.М. Полуботко. Опт. и спектр. 109, 4, 560 (2010).

[9] А.М. Полуботко. Опт. и спектр. 114, 5, 759 (2013).

[10] T. Lu, T.M. Cotton, R.L. Birke, J.R. Lombardi. Langmuir 5, 406 (1989).

[11] M.J. Frisch, G.W. Trucks, H.B. Schlegel, G.E. Scuseria. M.A. Robb, J.R. Cheeseman, J.A. Montgomery, jr., T. Vreven, K.N. Kudin, J.C. Burant, J.M. Millam, S.S. Iyengar, J. Tomasi, V. Barone, B. Mennucci, M. Cossi, G. Scalmani, N. Rega, G.A. Petersson, H. Nakatsuji, M. Hada, M. Ehara, K. Toyota, R. Fukuda, J. Hasegawa, M. Ishida, T. Nakajima, Y. Honda, O. Kitao, H. Nakai, M. Klene, X. Li, J.E. Knox, H.P. Hratchian, J.B. Cross, V. Bakken, C. Adamo, J. Jaramillo, R. Gomperts, R.E. Stratmann, O. Yazyev, A.J. Austin, R. Cammi, C. Pomelli, J.W. Ochterski, P.Y. Ayala, K. Morokuma, G.A. Voth, P. Salvador, J.J. Dannenberg, V.G. Zakrewski, S. Dapprich, A.D. Daniels, M.C. Strain, O. Farkas, D.K. Malick, A.D. Rabuck, K. Raghavachari, J.B. Foresman, J.V. Ortiz, Q. Cui, A.G. Baboul, S. Clifford, J. Cioslowski, B.B. Stefanov, G. Liu, A. Liashenko, P. Piskorz, I. Komaromi, R.L. Martin, D.J. Fox, T. Keith, M.A. Al-Laham, C.Y. Peng, A. Nanayakkara, M. Challacombe, P.M.W. Gill, B. Johnson, W. Chen, M.W. Wong, C. Gonzalez, J.A. Pople. Gaussiana 03. Revision E.01. Gaussian, Inc., Wallingford CT (2004).

[12] H. Kihara, Y. Gondo. J. Raman Spectrosc. 17, 263 (1986).

[13] I. Pockrand. Surface enhanced Raman vibrational studies at solid/gas interface. Springer Tracts in Modern Physics. Springer-Verlag, Berlin-Heidelberg-N. Y.-Tokyo. (1984). V. 104. 105 p.

[14] W.-H. Yang, J. Hulteen, G.C. Schatz, R.P. Van Duyne. J. Chem. Phys. 104, 4313 (1996). 